\title{
Offen und zugänglich
}

\section{Warum gerade nationale Fachzeitschriften nicht nur offenen Zugang brauchen, sondern auch Zugänglichkeit}

\author{
Caroline Fischer
}

Dieser Beitrag versteht sich als Ergänzung zur Diskussion über die Kluft zwischen internationalen und nationalen Fachzeitschriften, die Christoph Reichard' in dieser Zeitschrift aufgegriffen hat. Er plädiert für eine Stärkung deutschsprachiger Fachzeitschriften für die öffentliche Verwaltung. Dieser Artikel schließt sich diesem Plädoyer an und beleuchtet einen zusätzlichen Aspekt, der eine strategische Neuausrichtung nationaler Fachzeitschriften treiben kann: offener Zugang zu Publikationen (Open Access). Zugang wird in diesem Artikel in zwei Ebenen unterschieden: den freien Zugang zu Artikeln und Zugänglichkeit, im Sinne von Verständlichkeit und Nutzbarkeit für die Leser. fener Zugang wird gefordert, weil die öffentliche Hand Forschung sonst doppelt und dreifach finanziert: mit der Bezahlung der Wissenschaftlerinnen an sich, Förderung von Forschungsprojekten und dem „Rückkauf“ der Ergebnisse in Form von Artikeln oder Büchern. Der kostenfreie Zugang zu Forschungsergebnissen bezieht sich hier in der Regel auf Online-Publikationen, die durch (nicht kostenfreie) Druckversionen ergänzt werden können. Open Access ist in Zeitschriften weitaus häufiger verbreitet als bei Buchpublikationen ${ }^{2}$. Einige Zeitschriften publizieren vollständig offen, viele machen nur einzelne Artikel offen zugänglich.

Viele Wissenschaftlerinnen und Wissenschaftler publizieren heute lieber in hochrangig gerankten und damit häufig internationalen Fachzeitschriften. Nationale Fachzeitschriften haben immer stärkere Probleme, ausreichend (qualitativ hochwertige) Beiträge zu finden. Begründet liegt das in den „Karrieremetriken“ des Wissenschaftssystems. Gleichzeitig engagieren sich aber auch immer mehr Wissenschaftlerinnen und Wissenschaftler für offene Wissenschaft und wollen ihre Ergebnisse zielgruppengerecht kommunizieren. Die Wissenschaftskommunikation boomt wie nie. Diese Entwicklungen können nationale Fachzeitschriften ausnutzen und sich strategisch neu ausrichten, indem sie auf Open Access setzen und sich damit wieder attraktiver für Autorinnen machen. Eine solche Neuausrichtung sollte insbesondere der Fall sein, wenn es der eigene Anspruch ist, anwendungsorientiert zu sein sowie Praktikerinnen und Praktiker zu erreichen. Offener $\mathrm{Zu}$ gang muss dann mit Zugänglichkeit einhergehen. Beide Dimensionen von Open Access sollen im Folgenden umrissen und auf die Möglichkeiten für anwendungsorientierte (nationale) Zeitschriften hin diskutiert werden.

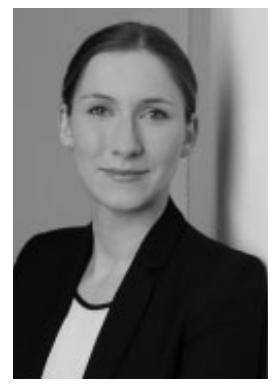

Caroline Fischer

Wissenschaftliche Mitarbeiterin am Lehrstuhl für Public und Nonprofit Management der Universität Potsdam.
Der offene $\mathrm{Zu}$ gang zu Publikationen wird im Rahmen offener Wissenschaft in den vergangenen Jahren immer stärker diskutiert und eingefordert. Of-

\section{Grüner und goldener Weg}

Open Access (OA) kann anhand der Kosten für die Autorinnen und des Zeitpunkts der Veröffentlichung unterschieden werden. Goldener OA bedeutet die offene Publikation des eigentlichen Artikels oder Buchs. Grüner OA hingegen meint, dass zusätzlich zur eigentlichen (geschlossenen) Publikation, ein Artikel oder Buch als pre- oder post-print ohne Zugangsbeschränkung veröffentlicht wird. Pre-print meint dabei eine Veröffentlichung vor Erscheinen eines Werks. Dies kann vor Peer-Review und Einreichung bei einer Zeitschrift geschehen oder aber erst, wenn ein Artikel den Feedbackprozess absolviert hat und zur Veröffentlichung angenommen ist. Dementsprechend unterscheidet sich hier häufig auch die Qualität der Beiträge. Für pre-prints existieren entsprechende Online-Portale, etwa SocArxiv ${ }^{3}$.

Wird ein Werk nach einer geschlossenen Veröffentlichung offen zweitveröffentlicht, ist von post-prints die Rede. Diese sind etwa in Schriftenreihen von Hochschulen und anderen Forschungseinrichtungen möglich. Für solche Zweitveröffentlichungen gelten häufig Embargozeiten, die die Verlage bei der Erstveröffentlichung festlegen und zwischen 12 und 24 Monaten schwanken. Dementsprechend verzögert sich grüner OA. Hin-

1 Reichard 2018

2 https://www.publisso.de/open-access-beraten/faqs/monografien-beitraegezu-sammelbaenden-und-konferenzbeitraege/.

3 https://osf.io/preprints/socarxiv. 
zu kommt immer stärker ein temporärer offener Zugang, etwa, wenn mit bereits erschienenen und häufig vielzitierten Artikeln virtuelle Themenhefte herausgegeben werden. Auch solche virtuellen Themenhefte sind für nationale Fachzeitschriften spannend, um Themensammlungen zu erzeugen, die für die Praxis Recherchen erleichtern können.

\section{Bezahlter und unbezahlter Open Access}

In so gut wie allen internationalen Journals ist es möglich, sich goldenen Open Access zu erkaufen. Die Gebühren hierfür Verlagen und wenigen hundert Euro bei kleineren Verlagen oder non-Profit-Journals (so genannte APCs - article processing charges). In letzterem Falle dienen diese beispielsweise der Finanzierung des Lektorats, in ersterem Falle sollen diese Gebühren die Einnahmeverluste ausgleichen, die durch den offenen Zugang entstehen. Es gibt aber durchaus auch Zeitschriften, die eine für die Autorinnen und Autoren kostenlose offene Publikation anbieschwanken zwischen 2000 und 3000 Euro bei kommerziellen

häufig mit einer stärkeren Nutzung einher. Etwa deuten Studien an, dass frei zugängliche Artikel häufiger zitiert werden, beispielsweise auch in der Politikwissenschaft ${ }^{4}$. Insofern kann offener Zugang auch die Wirkung einer Zeitschrift vergrößern.

Gerade für Fachzeitschriften, die sich an einen gemischten Leserkreis wenden, sind aber gerade nicht nur die Zitationen anderer Wissenschaftler von Bedeutung, sondern inwiefern die Publikationen Eingang in die Diskurse mit und unter den prakterinnen und Praktikern finden. Ich behaupte, dass dies mit offenem Zugang einfacher ist. Viele Verwaltungsakteure leisten sich keine Abos von Fachzeitschriften mehr, sondern recherchieren bei Fragen online. Wenn sie dort einen freien Zugang zu Fachzeitschriften haben, nutzen sie diese mit großer Wahrscheinlichkeit auch.

\section{Open Access in kleineren Wissenschaftsverlagen}

Gerade kleinere Verlage, die häufig nationale Fachzeitschriften verlegen, werden bisher aber weitestgehend von den Akteuren offener Wissenschaft übersehen. Die Allianz der deutschen Wissenschaftsorganisationen verhandelt Deals mit großen Verlagen, weil deren Abonnements hohe Kosten erzeugen. Eine Studie zu kleinen bis mittleren kommerziellen Wissenschaftsverlagen im deutschsprachigen Raum fand heraus, dass bei 59 Prozent der befragten Verlage die Nachfrage nach Open Access langsam und bei 31 Prozent sogar deutlich steigt ${ }^{5}$. Das gilt vor allem für Verlage, die mehr als zehn Zeitschriftentitel herausgeben. Auch bezüglich der Autorenakquise ist Open Access für die meisten befragten ten. Diese Zeitschriften sehen in der Regel Open Access für alle Beiträge vor und nicht nur, wenn es die Autorin oder der Autor explizit wünscht (und bezahlt).

\section{Stärker werdende Verpflichtung zu Open Access}

Die offene Publikation von Forschungsergebnissen wird, zurecht, immer stärker von Fördermittelgebern eingefordert oder gesetzlich geregelt. So haben sich etwa im Rahmen der „Coalition S“ viele nationale und internationale Forschungsförderer, wie die Europäische Kommission oder das britische, norwegische oder österreichische Äquivalent zur DFG vorgeschrieben, dass ab 2020 alle von ihnen geförderten Projekte offen publiziert werden müssen. In Dänemark beschloss das Wissenschaftsministerium eine entsprechende Richtlinie. Die niederländische Regierung hat sich vorgenommen, dass 2019 bereits 60 Prozent der Publikationen niederländischer Universitäten offen erfolgen müssen und 2024100 Prozent. Für Projekte im Rahmen von Horizont 2020 gilt die Pflicht zum Open Access schon jetzt. Die DFG fordert ihre Fördermittelempfänger zu einer offenen Publikation auf, will diese aber nicht verpflichtend einführen.

Diese Vorgaben der Fördermittelgeber können nationale Fachzeitschriften dazu nutzen, sich stärker als entsprechende Publikationsmöglichkeit zu positionieren. Solch ein offener Zugang geht
Verlage relevant. Jeder dritte Verlag geht außerdem davon aus, dass Open Access zum zukünftigen Standard des wissenschaftlichen Publizierens wird; weitere 60 Prozent sehen es als Ergänzung bestehender Verlagsangebote. Die Studie zeigt zudem auf, dass Verlage sich von Open Access eine höhere Sichtbarkeit und Wettbewerbsvorteile versprechen, aber auch, dass (noch) wenig Wissen über Geschäftsmodelle in diesem Bereich vorliegt.

\section{Was nichts kostet, ist nichts wert?}

Es herrscht in Bezug auf Open Access die Meinung, dass schlechte Artikel, die es woanders nicht geschafft haben, offen publiziert werden. Um einer solchen Tendenz entgegenzuwirken, ist eine intensive redaktionelle Auswahl und ein Review-System wichtig. Andernfalls befinden sich solche Zeitschriften in einem Teufelskreis: Wenn die mindere Qualität von Beiträgen ein schlechtes Image einer Zeitschrift erzeugt, werden dort keine oder immer weniger gute Beiträge eingereicht. Wenn aber der Zugang zu Artikeln offen ist und die Qualität stimmt, ist es fraglich, ob Leser tatsächlich lieber etwas für eine Fachzeitschrift bezahlen wollen würden.

4 Antelmann 2004; Harnad/Brody 2004.

5 Kaiser/Lackner 2018. 


\section{Offener Zugang heißt auch Zugänglichkeit}

Der freie Zugang zu Publikationen allein wird aber wahrscheinlich nicht dazu beitragen, (verwaltungs-)wissenschaftliche Forschung wieder stärker in den Diskurs mit der (Verwaltungs-)Praxis zu bringen. Vielmehr muss offener Zugang um Zugänglichkeit ergänzt werden. Dies betrifft sowohl Themen und Inhalte der Beiträge als auch deren Sprache.

Wenn sich nationale Fachzeitschriften an einen breiten Leserkreis richten wollen, müssen die Beiträge thematisch entsprechend ausgerichtet sein. Das wiederum kann nicht bedeuten, dass ein wissenschaftlicher Journalartikel mit elaborierter $\mathrm{Me}$ thodenbeschreibung in solchen Zeitschriften erscheint. Es kann aber auch nicht bedeuten, dass Akteure aus der Praxis bloße Pro- dass Wissenschaftlerinnen und Wissenschaftler ihre Arbeit wieder mehr auf Deutsch erklären müssen ${ }^{6}$. Nationale Fachzeitschriften können sich damit als Einstieg in ein wissenschaftliches Feld positionieren, nicht nur für Prakterinnene und Praktiker, sondern auch für Bürgerinnen und Bürger, Politikerinnen und Politiker oder Neulinge im Themenbereich.

Die eigenen Ergebnisse herunterzubrechen und zugänglich zu kommunizieren, ist freilich nicht einfach und nicht jede gute Wissenschaftlerin und jeder gute Wissenschaftler wird auch das gut beherrschen. Aber: zugängliches Formulieren kann geübt werden und beispielsweise auch im Team mit einer Autorin aus der Praxis geschehen. Zugängliches Publizieren sollte im Übrigen auch in der Grundlagenforschung oder Forschung zu anderen Länderkontexten erfolgen. Auch diese können verständlich für ein breites Publikum erklärt werden. Meiner Erfahrung nach ist Erklärbarkeit sogar ein Qualitätskriterium. Wer seine Forschung gut erklären kann, forscht häufig keinesfalls zu trivialen Themen, sondern hat die Materie gut durchdrungen.

National zu publizieren, widerspricht zunächst dem, was in vielen Disziplinen heute gewürdigt wird, sollte aber der Anspruch jeder Wissenschaftlerin und jedes Wissenschaftlers sein. Eine Publikationsstrategie, die den Anspruch der Anschlussfähigkeit in der Praxis und der Karrieremetriken gleichzeitig erfüllt, kann sein:

jektbeschreibungen publizieren. In beiden Fällen wird sich die jeweils andere Seite der Leserschaft nicht angesprochen fühlen. Die Kunst eine Fachzeitschrift sowohl für Wissenschaftlerinnen und Wissenschaftler als auch für Praktikerinnen und Praktiker attraktiv zu machen, liegt darin, Themen zu behandeln, die für beide Seiten relevant sind. Hierfür können Autorentandems nützlich sein. Ein erster Schritt in diese Richtung kann auch durch strukturierte Abstracts erfolgen, in denen explizit verlangt wird zu beschreiben, was der praktische und was der theoretische Beitrag eines Artikels ist. Hier sollten die Redaktionen entsprechende Standards setzen.

\section{Zugängliche Sprache}

Neben Themen, die einen breiten Leserkreis interessieren, ist es wichtig, diese verständlich aufzubereiten. Dieser Appell für zugängliche Sprache richtet sich an beide Autorengruppen anwendungsorientierter Fachzeitschriften. Für den verwaltungswissenschaftlichen Bereich etwa gilt dieser sowohl für Praktikerinnen und Praktiker, deren „Beamtensprache“ mitunter schwer zugänglich ist, wie auch für Wissenschaftlerinnen und Wissenschaftler, deren Fachsprache manches Unverständnis erzeugt.

Eine solche Zugänglichkeit kann nicht nur helfen, den Diskurs zwischen Verwaltungspraxis und -wissenschaft zu stärken, sondern auch das Vertrauen der Gesellschaft in die Wissenschaft und deren Ergebnisse zurückzugewinnen. Erst kürzlich forderte der langjährige Wissenschaftspolitiker Jürgen Zöllner zurecht, neueste Forschungsergebnisse zunächst international präsentieren und dabei einem Peer Review unterziehen und im Anschluss die Forschung herunterbrechen und national publizieren. Mit offenem Zugang und in zugänglicher Weise!

\section{Literatur}

Reichard, C. (2018): Die zunehmende Kluft zwischen nationalen und internationalen Fachzeitschriften, in: Verwaltung und Management $\mathrm{Nr}$. 6/2018, S. 295-298. https://doi.org/10.5771/0947-9856-2018-6-295

Antelmann, K. (2004): Do Open-Access Articles Have a Greater Research Impact?, in: College \& Research Libraries Nr. 5/2004, S. 372-382. https:// doi.org/10.586o/crl.65.5.372

Kaier, C./Lackner, K. (2018): Open Access aus der Sicht von Verlagen, in: BIBLIOTHEK - Forschung und Praxis. Advance online publication. https:// doi.org/10.18452/19635

Harnad, S./Brody, T. (2004): Comparing the Impact of Open Access (OA) vs. Non-OA Articles in the Same Journals, in: D-Lib Magazine Nr. 6/2004. doi:10.1045/june2004-harnad 